\title{
Asian Society for Gynecologic Robotic Surgery consen- sus guidelines on robotic surgery in gynecological cancer
}

\author{
Joseph S. $\mathrm{Ng}^{1}$, Mario M. Leitao ${ }^{2}$, Peter C. Lim ${ }^{3}$, Tae-Joong Kim", Sang Wun Kim ${ }^{5}$ \\ ${ }^{1}$ National University Cancer Institute, Singapore, Singapore; ${ }^{2}$ Memorial Sloan Kettering Cancer Center, New York, NY; ${ }^{3}$ Center of Hope, Reno, NV, \\ USA; ${ }^{4}$ Department of Obstetrics \& Gynecology, Samsung Medical Center, Seoul; ${ }^{5}$ Department of Obstetrics \& Gynecology, Yonsei University \\ College of Medicine, Seoul, Korea
}

This document outlines guidelines drafted by the Consensus Panel for Gynecologic Oncology of the Asian Society for Gynecologic Robotic Surgery (ASGRS) based on review of contemporaneous data on robotic surgery and related issues such as sentinel node biopsy and surgeon credentialing. These guidelines were also the basis of the Consensus Statement on robotic surgery in gynecologic oncology made at the Asian Summit on Robotic Surgery, October 2018.

Key Words: Clinical practice guideline; Robotic surgical procedures; Cervical cancer; Endometrial neoplasms; Ovarian neoplasms

\section{INTRODUCTION}

The use of robotic surgical technology in gynecology has seen an exponential increase globally since it was Food and Drug Administration-approved for gynecology in 2005. The primary reason driving this growth is the ease with which surgeons not traditionally laparoscopically trained are able to achieve proficiency and provide minimally invasive surgical care to women who require surgery. Consequently, robotic surgical technology has been shown to dramatically decrease the rates of laparotomy compared to traditional laparoscopy [1-3]. The Society for Gynecologic Oncology (SGO) Consensus Statement on Robotic Surgery published in 2012 outlined the basis for the use of robotic surgery (RS) in gynecological cancer surgery in a comprehensive document outlining the history, growth, and contemporary application and evidence for RS in each domain of women's cancer surgical care [4]. This document was drafted with the intention of updating the SGO Consensus Statement with a special emphasis on care models and practice patterns in gynecologic oncology in Asia. The intent is to provide a foundational document that is the basis for policy development, practice guidance, and clinical application for robotics in women's cancer surgical care in Asia.

This document covers the following domains: 1) Robotic

- Received: September 29, 2019 • Revised: October 21, 2019 •Accepted: October 23, 2019

- Correspondening author: Joseph S. Ng

National University Cancer Institute, 1E Kent Ridge Road, 119228, Singapore

E-mail: obgnsyj@nus.edu.sg

This is an Open Access article distributed under the terms of the Creative Commons Attribution Non-Commercial License (http://creativecommons.org/ licenses/by-nc/4.0) which permits unrestricted non-commercial use, distribution, and reproduction in any medium, provided the original work is properly cited. 
Surgery in Cervical Cancer; 2) Robotic Surgery in Endometrial Cancer; 3) Robotic Surgery in Ovarian Cancer; 4) Single-port Robotic Surgery in Gynecological Cancer; 5) The Role of Sentinel Lymph Node Biopsy in Robotic Surgery for Gynecological Cancer; and 6) Recommendations for Credentialing in Robotic Surgery in Gynecologic Oncology.

\section{ROBOTIC SURGERY IN CERVICAL CAN- CER}

This section seeks to update the data regarding the use of RS in cervical cancer since the SGO Consensus Statement on Robotic Surgery published in 2012 [4]. Additionally, this section will also highlight data from and reflect practice norms in Asia wherever appropriate.

Radical hysterectomy with surgical retroperitoneal nodal assessment is a key primary treatment modality in patients diagnosed with early stage cervical cancers. Nezhat and colleagues first reported on the use of laparoscopy to perform this procedure in 1992 [5]. Since then over 20 publications have compared laparoscopic (with and without robotic assistance) to laparotomy. All have noted improved perioperative outcomes without a concern for a compromised oncologic outcome.

The largest series comparing laparoscopic to open radical hysterectomy was reported by Nam and colleagues from Korea at a single center [6]. They retrospectively compared 263 laparoscopic to 263 open radical hysterectomies in patients with International Federation of Gynecology and Obstetrics (FIGO) stage IA2 to IIA cervical cancer. Laparoscopic radical hysterectomies were associated with significantly lower estimated blood loss, hospital stays and postoperative complications. The 5-year disease-free survival (DFS) was 93\% after laparoscopy and 94\% after laparotomy ( $P=$ non-significant). The 5-year overall survival was 95\% after laparoscopy and 96\% after laparotomy ( $P=$ non-significant).

Sert and colleagues reported the largest retrospective multi-center series comparing robotically-assisted compared to laparotomy for radical hysterectomy in patients with FIGO stage IA1 to IB2 cervical cancer [7]. They compared 259 cases performed with robotic platforms to 232 cases performed via laparotomy. Robotically-assisted procedures were associated with lower estimated blood loss, less intraoperative complications, lower transfusion rates, and shorter length of hospital stays. The recurrence rates were the same at $9 \%$ after both surgical approaches. A meta-analysis published in 2015 encompassing 26 publications further confirmed the benefit of robotics over laparotomy for radical hysterectomy [8].

Minimally invasive surgery (MIS) approaches, as compared to laparotomy, will result in decreased complications and lower length of stays which directly correlate to a significant reduction in overall costs of surgical care $[9,10]$. Scalici et al. [9] analyzed data from the American College of Surgeons NSQIP database and reported that over 8,000 less complications would occur and more than 500 million dollars would be saved annually if MIS were performed in $90 \%$ of all patients with uterine cancer. Our goal in surgery for gynecologic cancers should be to maximize MIS rates as it results in improved perioperative outcomes, lower healthcare costs on a societal basis without compromising oncologic outcomes. However, standard laparoscopic instrumentation has significant limitations which has led to very low and unacceptable rates of MIS in cervical cancer. Prior to introduction of the robotic platform, the rate of MIS for cervical cancer was less than 5\% in the United States [11]. The rate increased to approximately $30 \%$ in 2010 after 4 years of robotic platform use. A similar low rate of MIS for cervical cancer was noted in Korea [12]. The robotic platform provides significant advantages over standard laparoscopic instrumentation that greatly facilitates the ability to perform these complex procedures in cervical cancer with a MIS approach.

Recently, the results from the first ever completed randomized trial assessing MIS compared to laparotomy to perform radical hysterectomy for cervical cancer were presented by Dr. Ramirez at the Society of Gynecologic Oncology's Annual Meeting 2018 in New Orleans. The results of of the Laparoscopic versus Abdominal radical hysterectomy in early Cervical Cancer (LACC) trial have since been published [13] and are still being discussed and debated with results from newer trials looking specifically at robotic surgery in early cervical cancer, in progress. Therefore, we need to be cautious in how to interpret and apply these results broadly. Also, nearly $90 \%$ of the MIS procedures were done with conventional laparoscopic instrumentation without use of the robotic in the LACC trial. An invited editorial 
by Dr. Leitao has been published that provides additional thoughts regarding the presented results of the trial [14].

The Asian Consensus Guideline on Robotic Surgery in Cervical Cancer therefore recommends: 1) that a complete discussion with each patient is necessary regarding all available options in the surgical management of early cervical cancer. That each patient be made aware of the findings of recent randomized control trials that suggested poorer oncological outcomes were associated with MIS for early cervical cancer. This recommendation is STRONG based on general consensus and on the basis that this recommendation reflects clinical practice based on scientific validation and sound ethics. The quality of the evidence supporting this recommendation is STRONG [13]. And 2) that the findings of retrospective literature that support the view that MIS is equivalent to open surgery (OS) for cervical cancer should also be discussed with patients. This discussion should include the general advantages of MIS over OS which include but are not limited to lower blood loss, fewer inpatient days, and lower wound complication rates. This recommendation is STRONG based on consensus, and on the ethical principles of patient autonomy and full disclosure. The quality of the evidence supporting this recommendation is MODERATE [7-10].

\section{ROBOTIC SURGERY IN ENDOMETRIAL CANCER}

This section seeks to update the data regarding the use of RS in endometrial cancer since the SGO Consensus Statement on Robotic Surgery published in 2012 [4]. Additionally, this section will also highlight data from and reflect practice norms in Asia wherever appropriate.

Endometrial cancer is the most common gynecological malignancy in developed countries, and in Asia the incidence has increased over the past few decades [15]. The treatment of endometrial cancer involves a hysterectomy with bilateral salpingo-oophorectomy and surgical staging involving the removal of pelvic and or aortic lymph nodes to assess the full extent of disease spread. Prior to 1990, this procedure was accomplished via open laparotomy in a patient population that was often obese. The procedure was associated with significant morbidity from blood loss, organ injury, and prolonged hospitalization from wound complica- tions. In addition, the potential for long term complications resulting from incisional hernias, poor wound healing and thromboembolic events added to the healthcare cost of managing endometrial cancer. Thus, it was important to develop a minimally invasive surgical approach in treating these patients without compromising oncological outcomes.

MIS has revolutionized the management of gynecologic disorders over the last 40 years. However, the most substantial improvements have been made since the introduction of RS. The initial promise was that traditional laparoscopy would be a less invasive approach to hysterectomy, tubal ligation, adnexal surgery, and even more complex procedures such as lymphadenectomy and radical hysterectomy. The reality however is that since its introduction the uptake of traditional laparoscopy has been tepid as not all surgeons are naturally comfortable with the laparoscopic platform and physics which result in a steep and extended learning curve, and this naturally limits the number of patients that can benefit from it. In fact, the majority of advanced gynecologic surgeries are still being performed through an abdominal incision.

Dargent and Mathevet [16] first reported utilizing laparoscopic pelvic lymph node dissection (PLND) in conjunction with radical vaginal hysterectomy to treat cervical cancer. Shortly thereafter, Childers et al. [17] then demonstrated the feasibility of laparoscopic surgery (LS) in staging ovarian cancer. Since then, there has been an increase in the use of LS in staging women with uterine cancer [18]. The adoption of MIS in the management of endometrial cancer has been shown to have similar intraoperative complication rates, but with decreased postoperative morbidity and shorter hospitalization, with no difference in oncological outcomes [19]. In addition, LS also has been reported to improve quality of life [20]. However, LS has its drawbacks. GOG 2222 (GOG LAP2) showed a $26 \%$ conversion rate to OS due to poor visibility, and it was suggested that the conversion rate could potentially increase with increasing body mass index [19]. In addition, there are several other limitations to LS: the hand movements are counterintuitive, and the long instruments working through a fixed entry point cause small movements and even tremors to be accentuated. These factors make fine control difficult. Also, the instruments have a limited range of motion that often require ergonomi- 
cally challenging positions for the surgeon resulting in surgeon fatigue during lengthy or challenging cases, and therefore significantly degrading the safety of the procedure. Lastly, the 2-dimensional optics and the unstable camera platform result in loss of depth perception and difficult visualization, the success of the procedure therefore depends on the stability and skill of the assistant operating the camera. Due to these limitations, many complex surgical procedures such as surgical staging for endometrial cancer are still completed via OS. With the introduction of RS, many surgeries that would have been done with via OS are now being performed minimally invasively.

The current RS platform consists of three components: the surgeon's console, which directs the movements of the robotic arms, the vision system, and the patient-side cart, which in the latest system has four arms. After placement of port sites and docking the patient side cart, the surgeon sits at the console and is able to view the pelvis through a three-dimensional vision system in high definition. Furthermore, the camera system is stabilized by the robotic platform and easily controlled by the surgeon through foot pedals and arm movements. At the console, the surgeon controls the robotic arms and the instruments with natural hand and wrist motions that mimic movements performed in OS. The RS platform also reduces the effect of tremor. The surgeon has control of three robotic arms on which can be attached an array of powered cutting, sealing, and grasping instruments, in addition to the camera system. All of these components reduce the fatigue and strain that are experienced by laparoscopic surgeons during long or difficult cases.

Due to such advancements, the robotic platform allows less experienced laparoscopic surgeons to perform more complex procedures. The surgeon is able to progress quickly along the learning curve and accomplish tasks such as intra-corporeal suturing and knot tying, ureterolysis, lymphadenectomies, and lysis of dense adhesions with ease and improved visualization. Also, additional assistance can be achieved through the $10-15 \mathrm{~mm}$ assistant port to provide suctioning, retraction, vessel coaptation, passage of suture or laparotomy sponges, and even thrombogenic agents as needed. Furthermore, uterine manipulation by an assistant provides another method to improve visualization and access to the pelvis, which is unique to gynecologic surgery.
Hence, the learning curve is much shorter. In fact, the adoption of MIS was stagnant at about 15\% since the introduction of laparoscopic staging for endometrial cancer, however with the introduction of RS, the proportion of cases done minimally invasively for uterine cancer staging increased from $6.4 \%$ before robotics, to $80.5 \%$ in the third year after its introduction [21]. This rapid adoption in gynecologic oncology is thought to be attributed to the shorter learning curve and ease of use of the RS platform. When considering the delivery of surgical healthcare for endometrial cancer across entire institutions or health systems, it is worthwhile determining if open laparotomy is the most common mode of surgery, and how to efficiently convert all these cases to a minimally invasive approach. Mok et al. [22] showed that when the standard of care changed directly from open to RS for endometrial cancer, "outcomes traditionally associated with laparoscopic endometrial cancer staging" were achievable after relatively few cases by open surgeons leveraging surgical robotic technology.

Despite the adoption of RS in gynecologic oncology, there is only moderate-level evidence comparing its efficacy and safety to other traditional surgical approaches. Weinberg et al. [23] reported a comprehensive systematic review and meta-analysis comparing the efficacy of RS to OS and LS. In their comprehensive meta-analysis comparing RS to OS, operative times were significantly longer for RS with one study showing no difference [24]. Overall, there was a significant reduction in blood loss and duration of hospital stay in patients undergoing RS compared to OS. Lymph node assessment is an important part of the surgical staging of endometrial cancer. The adequacy of lymph node retrieval is therefore an important metric in the adequacy of the surgical approach. In their metanalysis, Boggess et al. [25] found no difference between RS and OS in retrieval of lymph nodes, with the exception of one study reported higher number of lymph node yields retrieved in the RS group. Lim et al. [26] reported fewer nodes in the RS group.

In a systematic review involving four meta-analyses of more than 6,300 patients of which 2,913 underwent robotic, 2,196 underwent laparoscopic, and 1,219 underwent OS for endometrial cancer, the results showed the lowest complication rates associated with robotic surgical staging. The RS group also had reduced operative blood loss and fewer conversions to laparotomy [27]. Most recently a pro- 
spective randomized trial also showed that RS was associated with shorter operative times with a lower conversion rate than LS, with similar oncological outcomes [28].

There is paucity of data for long-term cancer outcomes for OS and MIS in the treatment of endometrial cancer. Both the LAP2 and LACE studies, which were prospective randomized trials, demonstrated that the risk of cancer recurrence with laparoscopy versus laparotomy was small, and that the estimated 5-year overall survival was almost identical in both groups $[19,29]$. Currently there is no large randomized trial evaluating survival outcomes with robotassisted surgery however several retrospective studies comparing robot-assisted surgery with laparoscopy and laparotomy for the management of endometrial cancer found no differences in DFS and overall survival [30-32]. Cardenas-Goicoechea et al. [33] compared the survival analysis of robot-assisted versus traditional laparoscopic surgical staging for endometrial cancer and found no difference in DFS or overall survival between RS and LS while Park et al. [34] reported no difference in 3- and 5-year disease free survival between RS and OS in the treatment of endometrial cancer.

Currently, only the LAP2 trial and LACE trials provide level I data that demonstrates LS is not inferior to OS for treatment of endometrial cancer. There are no randomized prospective trials comparing LS, RS, and OS. The available literature has reported on MIS with either LS or RS compared to OS for endometrial cancer, with most reports citing MIS as feasible and associated with decreased blood loss, fewer complications, and shorter hospitalization albeit many of these studies are Canadian Task Force II levels of studies. The learning curve for RS appears to be shorter when compared to LS. Although the operative time is longer for MIS when compared to OS, many of these reported cases are experiences of surgeons early learning curve phases. As the surgeon gains more experience operative time naturally shortens [26]. Finally, with no discernible difference in cancer outcomes in 3- and 5-year overall survival, RS and LS must be considered equivalent options in the surgical management of endometrial cancer.

There have been multiple studies evaluating costs of MIS (RS and LS) to OS for the surgical treatment of endometrial cancer. It should be noted that comparative cost effectiveness analysis is highly complex. When considering the cost of procedures, many of the studies analyze the direct and indirect costs of the procedures without considering the downstream cost that is associated with the procedure. Bell et al. [35] analyzed the direct and indirect cost of OS, LS, and RS in the treatment of endometrial cancer. They found that the average cost for hysterectomy with staging for endometrial cancer via OS was most expensive compared to both RS and LS [35]. They attributed this cost to increased length of hospitalization in OS. There have been many other studies that have reported that the cost of RS is higher when compared to LS. They attributed the cost to longer operative time and disposables associated with the conduct of RS $[36,37]$. It should be noted that these studies involved small numbers of cases that were performed while the surgeons negotiated the RS learning curve. It has been suggested that the cost of robotic surgery decreases as the volume of surgery increases, which reflects the effect of proficiency and efficiency of the surgical team on overall cost [31]. Furthermore, when length of hospitalization and complications are considered, RS appears to be most costeffective [38].

The Asian Consensus Guideline on Robotic Surgery in Endometrial Cancer therefore states: 1) that MIS is equivalent to OS for the surgical treatment of endometrial cancer delivering equivalent oncological outcomes. Further, it is important to note that a significant number of the women who are diagnosed with endometrial cancer are obese. The surgical management of endometrial cancer should therefore take this into consideration. OS, especially in obese women, is associated with a higher rate of wound complications, prolonged recovery, and delays in initiating adjuvant treatment where necessary. This recommendation is STRONG based on general consensus in the context of contemporary clinical practice which is based on evidence that robotic surgery is at least equivalent to LS which has been associated with better short term outcomes compared to OS. The quality of the evidence for this recommendation is MODERATE $[19,23-29,33,34]$. 2) That when the cost of surgery for endometrial cancer is studied, minimally invasive approaches appear to be more cost-effective and the robotic surgical platform seems to increase the adoption of a minimally invasive approach. This is especially evident in the care of obese patients and in settings where surgical volume is high. This recommendation is STRONG 
based on general consensus in the context of contemporary clinical practice which is based evidence that robotic surgery is at least equivalent to LS which has been associated with better short term outcomes compared to OS. The quality of the evidence for this recommendation is MODERATE [19-22].

\section{ROBOTIC SURGERY IN OVARIAN CAN- CER}

This section seeks to update the data regarding the use of robotic surgery in ovarian cancer since the SGO Consensus Statement on Robotic Surgery published in 2012 [4]. Additionally, this section will also highlight data from and reflect practice norms in Asia wherever appropriate.

Ovarian and primary peritoneal cancers affect the entirety of the peritoneum and a basic surgical objective in ovarian cancer surgery is the thorough evaluation of all peritoneal surfaces. Only after such a thorough evaluation is made can the surgeon accurately assess the extent to which all gross disease has been removed. The growing importance of robotic surgery in gynecologic oncology is best illustrated by the series of longitudinal surveys conducted by the SGO in 2004, 2009, and 2015, which saw rates of robotic surgery utilization in grow from $27 \%$ in 2007 to $97 \%$ in 2015, and the role of MIS become a widely-adopted standard of care option in gynecologic oncology [39-41].

In the most comprehensive meta-analysis to date analyzing more than 3,200 patients, Cardenas-Goicoechea et al. [42] reviewed the feasibility of minimally invasive interval debulkling surgery after neoadjuvant chemotherapy in patients with FIGO stage IIIC-IV ovarian cancer. MIS referred to both robot-assisted and laparoscopic surgical approaches. Complete cytoreduction was achieved in almost $75 \%$ of patients who underwent minimally invasive interval cytoreductive surgery. This percentage of patients achieving optimal cytoreduction in both the open laparotomy and MIS groups was not significantly different. The authors concluded that in a highly-select group of patients with advanced ovarian cancer who had complete response or who were assessed to have a very low tumor burden prior to interval cytoreductive surgery, MIS remained a feasible option to achieve optimal cytoreduction. This conclusion is supported by findings from other series [43-45]. Further- more, Eriksson et al found that robotic surgery was associated with trend towards same day discharge whereas traditional laparoscopy was not [44]. Of note are the caveats made by the authors, that the biology and clinical behavior of ovarian cancer raises concerns over the adequacy of the exploration of the peritoneal cavity, especially the root of the bowel mesentery and the retroperitoneum, and therefore the completeness of the surgical debulking [42].

Gallotta et al. [46] suggested that in equally experienced hands, robotic surgery is significantly more efficient than laparoscopy in the staging of early ovarian cancer. While the authors suggest that current robotic systems allow multi-quadrant exploration and boast superior visualization, the data can only support the assertion that clinical outcomes in robotic surgery are non-inferior to laparosocopy [46].

Fagotti et al. [47] determined in their series that MIS which included robotic surgery, in conjunction with hyperthermic intra-peritoneal chemotherapy (HIPEC) in selected patients with isolated recurrent ovarian cancer was feasible, safe and resulted in less toxicity.

Magrina et al. [48] have been the only group to systematically compare the performance of open laparotomy, laparoscopy, and robotic surgery in ovarian cancer. In evaluating the role of robotic surgery in ovarian cancer in the primary and recurrent settings at their center, they concluded that laparoscopic and robotic surgical approaches were equivalent and preferable to open laparotomy when tumor excision alone or with one additional procedure was to be performed. In the setting of recurrent disease, they concluded that robotic surgery would be most effective in isolated disease in the diaphragms, liver, retro-rectal areas, and the pelvis and especially in cases where there was no need for redocking or additional trocars [48].

In selecting patients for MIS in recurrent ovarian cancer, Eriksson et al. [44] excluded patients if they had carcinomatosis, perihepatic disease, and tumor in inaccessible sites. Magrina et al. [49] maintain that patient selection remains the primary performance indicator in MIS for primary and recurrent ovarian cancer. Overall survival and progressionfree survival were related to the degree to which complete cytoreduction was achieved rather than the surgical route by which this state was achieved [49].

It appears that as robotic surgery has become an accept- 
ed standard of care option in other gynecological cancers such as cervical and endometrial cancer, its role in ovarian cancer has gained momentum over the years, especially with the ability of newer platforms to operate more readily in multiple anatomic compartments, and the prospect of novel robotic surgical platforms becoming commercially available in the next 5 years.

The Asian Consensus Guideline on Robotic Surgery in Ovarian cancer therefore makes the following recommendations: 1) that robotic surgery may be considered as an equivalent platform to LS in selected patients that demonstrate a complete response to neoadjuvant chemotherapy and only have isolated residual disease or have isolated recurrent ovarian cancer. This is a STRONG recommendation by consensus as it is a statement of the equivalence of all minimally invasive modalities in the surgical management of ovarian cancer. The quality of the evidence supporting the above recommendation is MODERATE $[39,42,43,45,46,48,49]$. 2) In selecting patients for robotic surgery, factors such as the presence of carcinomatosis, perihepatic metastases, and tumors in accessible sites might be considered. This is a STRONG recommendation by consensus as it reinforces basic surgical principles widely accepted in the community of women's cancer surgeons in the management of ovarian cancer. The quality of the evidence supporting the above recommendation is LOW $[17,47,49]$.

\section{SINGLE-PORT ROBOTIC SURGERY IN GYNECOLOGICAL CANCER}

This section seeks to introduce the data regarding singleport robotic surgery (SPRS) in gynecological cancer. This section will also highlight data from and reflect practice norms in Asia wherever appropriate.

Single-port surgery is a relatively new development in gynecological surgery with most of the development and expertise coming out of Asia. Among the potential benefits are aesthetic improvements, less pain, and higher patient satisfaction [50,51]. Specimen retrieval is also less cumbersome and less uncomfortable for the patient than through a laparotomy or mini-laparotomy incision. Nevertheless, poor ergonomics, instrument collision, and insufficient traction are hurdles unique to single-site LS. The SPRS platform compensates for the poor ergonomics and instrument colli- sions by providing stable 3D vision and managing the instruments, although the movements of the platform limit the non-robotic assistant's freedom within the operative field.

Vizza et al. [52] reported their experience of SPRS hysterectomy for low-risk endometrial cancer. Lymph node dissection was not necessary for this series. There were no conversions and no complication in all 15 cases, and the cuff was closed vaginally in all cases [52]. More recently, the same group reported operative outcomes on 20 radical hysterectomy and pelvic lymphadenectomy cases performed on the SPRS platform demonstrating feasibility of the completion of complex pelvic procedures [53].

PLND is feasible. Tateo et al. [54] presented one of the very first cases as a case report of robotic single-site pelvic lymphadenectomy in endometrial cancer. Yoo et al. [55] reported six cases of early gynecologic cancer where the surgeons successfully completed systematic pelvic lymphadenectomies in four cases and described the technique in detail. Yoon et al. [56] then demonstrated the feasibility of a hysterectomy, adnexectomy, and PLND on the SPRS platform. Yoo et al. [57] then went on to show the feasibility of para-aortic lympadenectomy on the SPRS platform by demonstrating this highly demanding procedure in a video clip of a staging operation for early stage ovarian cancer. Sentinel lymph node (SLN) mapping with SPRS platform has been reported to produce equivalent perioperative outcomes as multi-port robotic approaches while being more cost-effective [58].

Given the recent advent of single-site robotics, data on the overall value is still scarce. Corrado et al. [59] evaluated cosmetic outcome with a Patient and Observer Scar Assessment Score tool in 45 endometrial cancer patients. The authors concluded that an excellent cosmetic outcome with a high level of patient satisfaction could be expected with this approach [59].

The Asian Consensus Guideline on Single-port Robotic Surgery in Gynecological Oncology therefore makes the following recommendations: 1) that SPRS is be considered a feasible and reasonable option in the surgical treatment of low risk or early-stage endometrial and ovarian cancer, with the understanding that this approach is still in its infancy with limited adoption, and its value still undergoing validation. This strength of this recommendation is MODERATE 
based on small case series and case demonstrations. The quality of the evidence supporting this recommendation is LOW [52-57]. 2) That with the introduction of even more robotic platforms that support single-port surgery, performance data, oncological outcomes, and value-outcome based evaluations be systematically collected, analyzed, and shared to validate SPRS for gynecological cancers. The strength of this recommendation is STRONG based on expert consensus and the sound principles of systematic scientific validation. There is no quality statement associated with this recommendation based on an empiric recommendation by a panel of experts.

\section{THE ROLE OF SENTINEL LYMPH NODE BIOPSY AND ROBOTIC SURGERY IN GY- NECOLOGICAL CANCER}

This section seeks to introduce data on the role of sentinel lymph node biopsy in robotic surgery for gynecological cancers. This section will also highlight data from and reflect practice norms in Asia whereever appropriate.

SLN mapping or biopsy in the surgical staging of earlystage cervical cancer has demonstrated high sensitivity and significant negative predictive values [60]. While this technique has been used in tumors up to $4 \mathrm{~cm}$ in size, the best detection rates and mapping results are in cervical tumors less than $2 \mathrm{~cm}[61,62]$. The key to a successful SLN mapping is adherence to the SLN algorithm, which requires the performance of a side-specific nodal dissection in cases of failed mapping and removal of any suspicious or grossly enlarged nodes regardless of mapping. Ultrastaging of SLNS allows for higher detection of micrometastasis that may alter postoperative management [63].

In endometrial cancer, lymph node status affects staging, adjuvant treatment, and prognosis. Contemporary practice has changed based on multicenter trial data that suggests that lymphadenectomy may be safely omitted in grade 1 endometrioid tumors with less than 50\% myoinvasion [29,64]. The role SLN biopsy has become more prominent in the contemporary staging of endometrial cancer. By removing the highest yield lymph nodes, SLN biopsy promises equivalent diagnostic sensitivity without the morbidity of a full lymphadenectomy [65-67]. SLN biopsy has been validated in high-risk endometrial cancer and represents a rea- sonable alternative to complete systematic lymphadenectomy [68]. How et al. [69] reported that the overall recurrence free survival in patients that underwent SLN biopsy was same with systematic lymphadenectomy. Furthermore, patients who underwent SLN biopsy had lower pelvic side wall recurrence rates compared to those who had systematic lymphadenectomy [69].

The performance of SLN identification with robotic fluorescence imaging in the detection of metastatic endometrial and cervical cancer was evaluated in the FIRES trial. This large prospective, multicenter trial studied the role and diagnostic accuracy of SLN biopsy for endometrial and cervical cancer. Results of this trial reported a sensitivity of 97.2\% and a false negative rate of $3 \%$ overall. The FIRES trial concluded that and SLN biopsy has an acceptable diagnostic accuracy in detecting lymphatic metastases, and can replace lymphadenectomy for this diagnostic purpose in cervical and endometrial cancer [67].

The Asian Consensus Guideline on Sentinel Lymph Node Biopsy in Robotic Surgery for gynecological cancers therefore states: 1) that SLN biopsy algorithms are a reasonable diagnostic alternative to systematic lymphadenectomy in the robotic surgical management of endometrial and cervical cancer. This recommendation is STRONG based on current clinical practice trends to reduce the morbidity of nodal assessment without sacrificing diagnostic accuracy. The quality of the evidence supporting this statement is MODERATE $[60,61,64,65,67-69]$. 2) That specific recommendations include the removal of all mapped nodes, all grossly enlarged or clinically-suspicious lymph nodes, and a sidespecific lymphadenectomy in instances where mapping fails. The strength of this recommendation is STRONG based on clinical consensus and existing validated algorithms guiding sentinel node biopsy in gynecological cancer. The quality of the evidence supporting this statement is MODERATE [60-69].

\section{RECOMMENDATIONS FOR CREDEN- TIALING IN ROBOTIC SURGERY IN GY- NECOLOGIC ONCOLOGY}

The recommendations in this document will mirror other documents outlining the credentialing process in robotic surgery published and supported by the Asian Society for 
Gynecologic Robotic Surgery, the ASGRS.

The requirements for credentialing are as follows:

1) credentialed by the institution or health system to perform the open procedure.

1-1) This first requirement establishes the surgeon's proficiency and track record in being able to safely perform the procedure via laparotomy which indicates:

\section{1-1-1) an implicit knowledge of surgical anatomy}

1-1-2) a firm grasp of surgical oncological principles surrounding the procedure

1-2) This requirement also establishes the necessary skill sets that surgeons will replicate robotically which will allow them to optimize the advantages of the robotic surgical platform which in turn translates to a safer, more precise procedure.

2) Proof of a basic understanding of the robotic surgical platform and its operation. This basic understanding usually includes but is not necessarily limited to the process by which the system interfaces with the patient (docking), key safety features built into the system, basic troubleshooting, basic system setup, and available instrumentation. This requirement is usually fulfilled through completion of the daVinci Basic Systems Training or an equivalent.

3) Performance of procedures under guidance or proctoring. The usual minimum requirement is that FIVE procedures be performed by the surgeon seeking credentialing under the direct supervision of a surgeon credentialed to perform the robotic procedure or under the supervision of a surgeon recognized by the community to be an expert in the procedure.

4) That some form of continuing assessment be put in place to ensure that only surgeons that have a steady and consistent surgical case load continue to be credentialed. Surgeons that fall below that caseload threshold should either be proctored when operating or have periodic skills maintenance through simulation training till caseloads rise above the established institutional threshold.

The Asian Consensus Guideline therefore states that the above recommendations be used to develop requirements that are specific and relevant to the individual healthcare environments throughout Asia with the primary objective of protecting patient safety and a secondary objective of supporting the growth of an efficient robotic surgical service.

\section{Conflict of interest}

No potential conflict of interest relevant to this article was reported.

\section{Acknowledgments}

The authors acknowledge the academic and collegial support of the following in providing their input and sharing from their wealth of experience which has helped the Consensus Panel to develop these guidelines. The Consensus Panel therefore acknowledges: Ji-Heum Paek (Ajou University), Keun-Ho Lee (The Catholic University of Korea), Chi-Heum Cho (Keimyung University), Keiichi Isaka (Tokyo Medical University), Hextan Ngan (Hong Kong University), Kung-Liangh Wang (Mackay Hospital, Taiwan), Jeffrey Low (National University of Singapore).

\section{References}

1. Bogani G, Multinu F, Dowdy SC, Cliby WA, Wilson TO, Gostout $\mathrm{BS}$, et al. Incorporating robotic-assisted surgery for endometrial cancer staging: analysis of morbidity and costs. Gynecol Oncol 2016;141:218-24.

2. Leitao MM, Narain WR, Boccamazzo D, Sioulas V, Cassella D, Ducie JA, et al. Impact of robotic platforms on surgical approach and costs in the management of morbidly obese patients with Newly Diagnosed Uterine Cancer. Ann Surg Oncol 2016;23:2192-8

3. Mäenpää MM, Nieminen K, Tomás El, Laurila M, Luukkaala TH, Mäenpää JU. Robotic-assisted vs traditional laparoscopic surgery for endometrial cancer: a randomized controlled trial. Am J Obstet Gynecol 2016;215:588.e1-588.e7.

4. Ramirez PT, Adams S, Boggess JF, Burke WM, Frumovitz MM, Gardner GJ, et al. Robotic-assisted surgery in gynecologic oncology: a Society of Gynecologic Oncology consensus statement. Developed by the Society of Gynecologic Oncology's Clinical Practice Robotics Task Force. Gynecol Oncol 2012;124:180-4.

5. Nezhat CR, Burrell MO, Nezhat FR, Benigno BB, Welander CE. Laparoscopic radical hysterectomy with paraaortic and pelvic node dissection. Am J Obstet Gynecol 1992;166:864-5.

6. Nam JH, Park JY, Kim DY, Kim JH, Kim YM, Kim YT. Laparoscopic versus open radical hysterectomy in early-stage cervical cancer: long-term survival outcomes in a matched cohort study. Ann Oncol 2012;23:903-11.

7. Sert BM, Boggess JF, Ahmad S, Jackson AL, Stavitzski NM, Dahl AA, et al. Robot-assisted versus open radical hysterec- 
tomy: a multi-institutional experience for early-stage cervical cancer. Eur J Surg Oncol 2016;42:513-22.

8. Shazly SA, Murad MH, Dowdy SC, Gostout BS, Famuyide AO. Robotic radical hysterectomy in early stage cervical cancer: a systematic review and meta-analysis. Gynecol Oncol 2015;138:457-71.

9. Scalici J, Laughlin BB, Finan MA, Wang B, Rocconi RP. The trend towards minimally invasive surgery (MIS) for endometrial cancer: an ACS-NSQIP evaluation of surgical outcomes. Gynecol Oncol 2015;136:512-5.

10. Xu T, Hutfless SM, Cooper MA, Zhou M, Massie AB, Makary MA. Hospital cost implications of increased use of minimally invasive surgery. JAMA Surg 2015;150:489-90.

11. Wright JD, Herzog TJ, Neugut AI, Burke WM, Lu YS, Lewin SN, et al. Comparative effectiveness of minimally invasive and abdominal radical hysterectomy for cervical cancer. Gynecol Oncol 2012;127:11-7.

12. Lee EJ, Park HM. Trends in laparoscopic surgery for hysterectomy in Korea between 2007 and 2009. J Obstet Gynaecol Res 2014;40:1695-9.

13. Ramirez PT, Frumovitz M, Pareja R, Lopez A, Vieira M, Ribeiro $\mathrm{R}$, et al. Minimally invasive versus abdominal radical hysterectomy for cervical cancer. N Engl J Med 2018;379:1895-904.

14. Leitao MM Jr. The LACC trial: has minimally invasive surgery for early-stage cervical cancer been dealt a knockout punch? Int J Gynecol Cancer 2018;28:1248-50.

15. Lortet-Tieulent J, Ferlay J, Bray F, Jemal A. International patterns and trends in endometrial cancer incidence, 1978-2013. J Natl Cancer Inst 2018;110:354-61.

16. Dargent D, Mathevet P. Mathevet, Schauta's vaginal hysterectomy combined with laparoscopic lymphadenectomy. Baillieres Clin Obstet Gynaecol 1995;9:691-705.

17. Childers JM, Lang J, Surwit EA, Hatch KD. Laparoscopic surgical staging of ovarian cancer. Gynecol Oncol 1995;59:25-33.

18. Barakat RR, Lev G, Hummer AJ, Sonoda Y, Chi DS, Alektiar $\mathrm{KM}$, et al. Twelve-year experience in the management of endometrial cancer: a change in surgical and postoperative radiation approaches. Gynecol Oncol 2007;105:150-6.

19. Walker JL, Piedmonte MR, Spirtos NM, Eisenkop SM, Schlaerth JB, Mannel RS, et al. Laparoscopy compared with laparotomy for comprehensive surgical staging of uterine cancer: Gynecologic Oncology Group Study LAP2. J Clin Oncol 2009;27:5331-6.

20. Kornblith AB, Huang HQ, Walker JL, Spirtos NM, Rotmensch J, Cella D. Quality of life of patients with endometrial cancer undergoing laparoscopic international federation of gynecology and obstetrics staging compared with laparotomy: a Gynecologic Oncology Group study. J Clin Oncol 2009;27:5337-42.

21. Paley PJ, Veljovich DS, Shah CA, Everett EN, Bondurant AE, Drescher CW, et al. Surgical outcomes in gynecologic oncology in the era of robotics: analysis of first 1000 cases. Am J Obstet
Gynecol 2011;204:551.e1-9.

22. Mok ZW, Yong EL, Low JJ, Ng JS. Clinical outcomes in endometrial cancer care when the standard of care shifts from open surgery to robotics. Int J Gynecol Cancer 2012;22:819-25.

23. Weinberg L, Rao S, Escobar PF. Escobar, Robotic surgery in gynecology: an updated systematic review. Obstet Gynecol Int 2011;2011:852061.

24. Jung YW, Lee DW, Kim SW, Nam EJ, Kim JH, Kim JW, et al. Robot-assisted staging using three robotic arms for endometrial cancer: comparison to laparoscopy and laparotomy at a single institution. J Surg Oncol 2010;101:116-21.

25. Boggess JF, Gehrig PA, Cantrell L, Shafer A, Ridgway M, Skinner EN, et al. A comparative study of 3 surgical methods for hysterectomy with staging for endometrial cancer: robotic assistance, laparoscopy, laparotomy. Am J Obstet Gynecol 2008;199:360. e1-9.

26. Lim PC, Kang E, Park DH. A comparative detail analysis of the learning curve and surgical outcome for robotic hysterectomy with lymphadenectomy versus laparoscopic hysterectomy with lymphadenectomy in treatment of endometrial cancer: a case-matched controlled study of the first one hundred twenty two patients. Gynecol Oncol 2011;120:413-8.

27. Rabinovich A. Minimally invasive surgery for endometrial cancer: a comprehensive review. Arch Gynecol Obstet 2015;291:721-7.

28. Cardenas-Goicoechea J, Adams S, Bhat SB, Randall TC. Surgical outcomes of robotic-assisted surgical staging for endometrial cancer are equivalent to traditional laparoscopic staging at a minimally invasive surgical center. Gynecol Oncol 2010;117:224-8.

29. Janda M, Gebski V, Davies LC, Forder P, Brand A, Hogg R, et al. Effect of total laparoscopic hysterectomy vs total abdominal hysterectomy on disease-free survival among women with stage I endometrial cancer: a randomized clinical trial. JAMA 2017;317:1224-33.

30. Chiou HY, Chiu LH, Chen CH, Yen YK, Chang CW, Liu WM. Comparing robotic surgery with laparoscopy and laparotomy for endometrial cancer management: a cohort study. Int J Surg 2015;13:17-22.

31. Coronado PJ, Herraiz MA, Magrina JF, Fasero M, Vidart JA. Comparison of perioperative outcomes and cost of roboticassisted laparoscopy, laparoscopy and laparotomy for endometrial cancer. Eur J Obstet Gynecol Reprod Biol 2012;165:28994.

32. Corrado G, Cutillo G, Pomati G, Mancini E, Sperduti I, Patrizi L, et al. Surgical and oncological outcome of robotic surgery compared to laparoscopic and abdominal surgery in the management of endometrial cancer. Eur J Surg Oncol 2015;41:107481.

33. Cardenas-Goicoechea J, Shepherd A, Momeni M, Mandeli J, Chuang L, Gretz H, et al. Survival analysis of robotic versus 
traditional laparoscopic surgical staging for endometrial cancer. Am J Obstet Gynecol 2014;210:160.e1-160.e11.

34. Park HK, Helenowski IB, Berry E, Lurain JR, Neubauer NL. A comparison of survival and recurrence outcomes in patients with endometrial cancer undergoing robotic versus open surgery. J Minim Invasive Gynecol 2015;22:961-7.

35. Bell MC, Torgerson J, Seshadri-Kreaden U, Suttle AW, Hunt S. Comparison of outcomes and cost for endometrial cancer staging via traditional laparotomy, standard laparoscopy and robotic techniques. Gynecol Oncol 2008;111:407-11.

36. Holtz DO, Miroshnichenko G, Finnegan MO, Chernick M, Dunton CJ. Endometrial cancer surgery costs: robot vs laparoscopy. J Minim Invasive Gynecol 2010;17:500-3.

37. Wright JD, Burke WM, Wilde ET, Lewin SN, Charles AS, Kim $\mathrm{JH}$, et al. Comparative effectiveness of robotic versus laparoscopic hysterectomy for endometrial cancer. J Clin Oncol 2012;30:783-91.

38. Barnett JC, Judd JP, Wu JM, Scales CD Jr, Myers ER, Havrilesky LJ. Cost comparison among robotic, laparoscopic, and open hysterectomy for endometrial cancer. Obstet Gynecol 2010;116:685-93

39. Conrad LB, Ramirez PT, Burke W, Naumann RW, Ring KL, Munsell MF, et al. Role of minimally invasive surgery in gynecologic oncology: an updated survey of members of the Society of Gynecologic Oncology. Int J Gynecol Cancer 2015;25:1121-7.

40. Frumovitz M, Ramirez PT, Greer M, Gregurich MA, Wolf J, Bodurka DC, et al. Laparoscopic training and practice in gynecologic oncology among Society of Gynecologic Oncologists members and fellows-in-training. Gynecol Oncol 2004;94:74653.

41. Mabrouk M, Frumovitz M, Greer M, Sharma S, Schmeler KM, Soliman PT, et al. Trends in laparoscopic and robotic surgery among gynecologic oncologists: a survey update. Gynecol Oncol 2009;112:501-5.

42. Cardenas-Goicoechea J, Wang Y, McGorray S, Saleem MD, Carbajal Mamani SL, Pomputius AF, et al. Minimally invasive interval cytoreductive surgery in ovarian cancer: systematic review and meta-analysis. J Robot Surg 2019;13:23-33.

43. Ackroyd SA, Thomas S, Angel C, Moore R, Meacham PJ, DuBeshter B. Interval robotic cytoreduction following neoadjuvant chemotherapy in advanced ovarian cancer. J Robot Surg 2018;12:245-50.

44. Eriksson AGZ, Graul A, Yu MC, Halko A, Chi DS, Zivanovic O, et al. Minimal access surgery compared to laparotomy for secondary surgical cytoreduction in patients with recurrent ovarian carcinoma: perioperative and oncologic outcomes. Gynecol Oncol 2017;146:263-7.

45. Onda T, Satoh T, Saito T, Kasamatsu T, Nakanishi T, Nakamura K, et al. Comparison of treatment invasiveness between upfront debulking surgery versus interval debulking surgery following neoadjuvant chemotherapy for stage III/IV ovarian, tubal, and peritoneal cancers in a phase III randomised trial: Japan Clinical Oncology Group Study JCOG0602. Eur J Cancer 2016;64:2231.

46. Gallotta V, Cicero C, Conte C, Vizzielli G, Petrillo M, Fagotti A, et al. Robotic versus laparoscopic staging for early ovarian cancer: a case-matched control study. J Minim Invasive Gynecol 2017;24:293-8.

47. Fagotti A, Petrillo M, Costantini B, Fanfani F, Gallotta V, Chiantera $\mathrm{V}$, et al. Minimally invasive secondary cytoreduction plus HIPEC for recurrent ovarian cancer: a case series. Gynecol Oncol 2014;132:303-6.

48. Magrina JF, Zanagnolo V, Noble BN, Kho RM, Magtibay P. Robotic approach for ovarian cancer: perioperative and survival results and comparison with laparoscopy and laparotomy. Gynecol Oncol 2011;121:100-5.

49. Magrina JF, Cetta RL, Chang YH, Guevara G, Magtibay PM. Analysis of secondary cytoreduction for recurrent ovarian cancer by robotics, laparoscopy and laparotomy. Gynecol Oncol 2013;129:336-40.

50. Jung YW, Kim SW, Kim YT. Recent advances of robotic surgery and single port laparoscopy in gynecologic oncology. J Gynecol Oncol 2009;20:137-44

51. Uppal S, Frumovitz M, Escobar P, Ramirez PT. Laparoendoscopic single-site surgery in gynecology: review of literature and available technology. J Minim Invasive Gynecol 2011:18:12-23

52. Vizza E, Corrado G, Mancini E, Baiocco E, Patrizi L, Fabrizi L, et al. Robotic single-site hysterectomy in low risk endometrial cancer: a pilot study. Ann Surg Oncol 2013;20:2759-64.

53. Vizza E, Chiofalo B, Cutillo G, Mancini E, Baiocco E, Zampa A, et al. Robotic single site radical hysterectomy plus pelvic lymphadenectomy in gynecological cancers. J Gynecol Oncol 2018;29:e2

54. Tateo S, Nozza A, Del Pezzo C, Mereu L. Robotic single-site pelvic lymphadenectomy. Gynecol Oncol 2014;134:631.

55. Yoo HN, Kim TJ, Lee YY, Choi CH, Lee JW, Bae DS, et al. Singlesite robotic surgery in gynecologic cancer: a pilot study. J Gynecol Oncol 2015;26:62-7.

56. Yoon A, Yoo HN, Lee YY, Lee JW, Kim BG, Bae DS, et al. Robotic single-port hysterectomy, adnexectomy, and lymphadenectomy in endometrial cancer. J Minim Invasive Gynecol 2015;22:322

57. Yoo JG, Kim WJ, Lee KH. Single-site robot-assisted laparoscopic staging surgery for presumed clinically early-stage ovarian cancer. J Minim Invasive Gynecol 2018;25:380-1.

58. Moukarzel LA, Sinno AK, Fader AN, Tanner EJ. Comparing Single-site and multiport robotic hysterectomy with sentinel lymph node mapping for endometrial cancer: surgical outcomes and cost analysis. J Minim Invasive Gynecol 2017;24:977-83.

59. Corrado G, Calagna G, Cutillo G, Insinga S, Mancini E, Baiocco E, et al. The patient and observer scar assessment scale to 
evaluate the cosmetic outcomes of the robotic single-site hysterectomy in endometrial cancer. Int J Gynecol Cancer 2018;28:194-9.

60. Salvo G, Ramirez PT, Levenback CF, Munsell MF, Euscher ED, Soliman PT, et al. Sensitivity and negative predictive value for sentinel lymph node biopsy in women with early-stage cervical cancer. Gynecol Oncol 2017;145:96-101.

61. Bats AS, Mathevet P, Buenerd A, Orliaguet I, Mery E, Zerdoud $S$, et al. The sentinel node technique detects unexpected drainage pathways and allows nodal ultrastaging in early cervical cancer: insights from the multicenter prospective SENTICOL study. Ann Surg Oncol 2013;20:413-22.

62. Kim JH, Kim DY, Suh DS, Kim JH, Kim YM, Kim YT, et al. The efficacy of sentinel lymph node mapping with indocyanine green in cervical cancer. World J Surg Oncol 2018;16:52.

63. Cormier B, Diaz JP, Shih K, Sampson RM, Sonoda Y, Park KJ, et al. Establishing a sentinel lymph node mapping algorithm for the treatment of early cervical cancer. Gynecol Oncol 2011;122:275-80.

64. ASTEC study group, Kitchener H, Swart AM, Qian Q, Amos C, Parmar MK. Efficacy of systematic pelvic lymphadenectomy in endometrial cancer (MRC ASTEC trial): a randomised study.
Lancet 2009;373:125-36.

65. Ballester M, Dubernard G, Lécuru F, Heitz D, Mathevet P, Marret $\mathrm{H}$, et al. Detection rate and diagnostic accuracy of sentinelnode biopsy in early stage endometrial cancer: a prospective multicentre study (SENTI-ENDO). Lancet Oncol 2011;12:46976.

66. Geppert B, Lönnerfors C, Bollino M, Persson J. Sentinel lymph node biopsy in endometrial cancer-feasibility, safety and lymphatic complications. Gynecol Oncol 2018;148:491-8.

67. Rossi EC, Kowalski LD, Scalici J, Cantrell L, Schuler K, Hanna $R K$, et al. A comparison of sentinel lymph node biopsy to lymphadenectomy for endometrial cancer staging (FIRES trial): a multicentre, prospective, cohort study. Lancet Oncol 2017;18:384-92.

68. Soliman PT, Westin SN, Dioun S, Sun CC, Euscher E, Munsell MF, et al. A prospective validation study of sentinel lymph node mapping for high-risk endometrial cancer. Gynecol Oncol 2017;146:234-9.

69. How J, Gauthier C, Abitbol J, Lau S, Salvador S, Gotlieb R, et al. Impact of sentinel lymph node mapping on recurrence patterns in endometrial cancer. Gynecol Oncol 2017;144:503-9. 